\title{
The Use of Tele Practice in Assessment of Middle Ear Function in Adults Living with HIV During the COVID-19 Pandemic
}

\author{
Ben Sebothoma $^{1}$ (D) Katijah Khoza-Shangase $^{1}$ (I) ${\text { Dipuo } \text { Masege }^{2} \text { (i) }}^{\cdot}$ \\ Duane $\mathrm{Mol}^{3}$ (I)
}

Received: 10 August 2021/Accepted: 21 August 2021/Published online: 5 September 2021

(C) Association of Otolaryngologists of India 2021

\begin{abstract}
Coronavirus disease (COVID-19) pandemic is the latest threat to global health that causes severe acute respiratory syndrome (SARS). Tele-practice has inadvertently sprung to the forefront to become a common practice amongst healthcare providers during COVID-19. Limited evidence exists on the use of tele-practice in assessing middle ear function in adults living with HIV during the COVID-19 pandemic. The aims of this study were to investigate the use of tele-practice for assessment of middle ear function in adults with HIV during the COVID-19 pandemic. A quantitative observational, cross-sectional design was adopted. A total of 134 adults diagnosed with HIV were purposively selected from the HIV clinic. An audiology researcher, in the role of site-facilitator, captured video otoscopic images of the tympanic membrane using a video otoscopy for all participants through asynchronous tele-practice. All captured images were sent to two
\end{abstract}

Ben Sebothoma

Ben.Sebothoma@wits.ac.za

Katijah Khoza-Shangase

Katijah.Khoza-Shangase@wits.ac.za

Dipuo Masege

dipuo.masege@wits.ac.za

Duane Mol

duanemol@netactive.co.za

1 Department of Speech Pathology and Audiology, School of Human and Community Development, Faculty of

Humanities, University of the Witwatersrand, Private bag X3, Wits, Johannesburg 2050, South Africa

2 Department of Otorhinolaryngology Head and Neck Surgery, Baragwanath Academic Hospital, Johannesburg, South Africa

3 Department of Otorhinolaryngology Head and Surgery, Ear and Eye Clinic, Alberton, South Africa independent otorhinolaryngologists for diagnosis. Findings of this study indicated that tele-practice can be used to assess middle ear function in adults living with HIV during COVID-19 pandemic. When asynchronous tele-practice was used, there was a moderate diagnostic agreement $(\mathrm{k}=0.58)$ between the two otorhinolaryngologists on abnormality versus normality, but poor agreement $(\mathrm{k}=0.15)$ on the nature of abnormality (e.g. OME vs CSOM). Current findings highlight the urgent need for a widespread use of tele-practice during the continued clinical follow up and management of adults living with HIV, and the implementation of tele-practice, particularly in low- and middle-income countries (LMICs) where capacity versus demand challenges related to ear and hearing care continue to exists.

Keywords Adults · HIV · Middle ear assessment . Tele-practice

\section{Introduction}

The Human Immunodeficiency virus (HIV) remains one of the biggest public health problems, affecting millions of people globally [1]. There was an estimated 36.9 million [31.1million -43.9 million] people living with HIV globally in 2018, with adult population accounting for $95 \%$ of the pandemic [1]. The high prevalence of HIV has been reported to be greater in low and middle income countries (LMICs), where South Africa is located [2]. Recent data indicates that the prevalence of HIV in South Africa is 7.5 million, an approximately $20 \%$ of the global population [2]. Although the antiretroviral (ART) regimen has been shown to be an effective treatment, HIV continues to 
increase the risk of otological and audiological impairments [3-6].

Middle ear pathologies are among the common otological manifestations of HIV [7, 8], with prevalence rates reaching approximately $60 \%$ [9]. Middle ear pathologies are characterized primarily by a dysfunction of the Eustachian tube due to weakened immune system [10], leading to unprevented pathogens, causing an accumulation of fluid in the middle ear cavity, and lack of vibratory movement of the tympanic membrane and ossicular chain [11]. Given the persistence of middle ear pathologies in this population, early identification and intervention must be implemented in order to prevent the potential long-term impacts that may result from late or lack of treatment [12-14]. Sebothoma and Khoza-Shangase [15] argue for increased efforts towards preventive care for middle ear pathologies through programmatic approaches that are delivered via innovative hybrid service delivery models, such as telepractice combined with task-shifting, within the South African context.

Traditionally, middle ear pathologies are identified by audiologists and otorhinolaryngologist using acoustic immittance measures and otomicroscopy respectively $[16,17]$. These measures are often used in direct close contact with patients presenting with audiological or/and otological symptoms. However, due to the COVID-19 pandemic, and the fact that examination around the ear, nose and throat has been reported to present the highest risk of COVID-19 transmission [18], these traditional methods became challenging to use, over and above the challenges of healthcare priorities re-arrangement brought on by COVID-19. Furthermore, the Centers for Disease Control and Prevention (CDC) suggest that individuals with HIV might be at an increased risk for developing severe illnesses due to COVID-19 [19]. Therefore, tele-practice became an urgent alternative tool for healthcare service delivery due to its remoteness and its ability to reduce close contact between patients and health care providers, thus preventing the spread of the virus [20, 21]. Tele-practice involves the use of technology to remotely assess and manage patients [22].

Although some otorhinolaryngologists have shared their challenges in using tele-practice during COVID-19 pandemic [23, 24], governmental health and safety regulations have necessitated the use of non-contact methods to provide healthcare services. Few research studies on the use of tele-practice have found the benefits of using this method during COVID-19 pandemic [25, 26], however, limited published evidence exists on the use of tele-practice in the assessment of middle ear function in adults living with HIV during COVID-19, particularly in LMICs where the infrastructure for tele-practice is limited [27] and resources to deal with undetected and untreated middle ear pathologies is extremely limited [28]. Therefore, the purpose of this study was to investigate the use of tele-practice in the assessment of middle ear function in adults living with HIV during the COVID-19 pandemic.

\section{Specific Objectives}

1. To describe middle ear function of adults living with HIV as diagnosed by otorhinolaryngologists using asynchronous tele-practice.

2. To determine reliability of using tele-practice to assess middle ear function during the COVID-19 pandemic.

\section{Methods}

This study employed a quantitative, observational, crosssectional design [29]. A non-probability purposive sampling method was used to recruit and select participants who met the inclusion criteria. Participants recruited and included in the study were adults aged 18 years and older diagnosed with HIV and attending HIV clinic. Exclusion criteria included adults who presented with otorrhea on the day of testing [30]. The study was carried out from September 2020 to December 2020 after receiving ethical approval from the Human Research Ethics Committee (Medical) of the University of the Witwatersrand: M190752. This was the COVID-19 lockdown period when face-to-face consultations at the otorhinolaryngology department at the tertiary hospital were postponed because of the infrastructural challenges, which do not permit social distancing. Therefore, service provision was restricted to patients requiring emergency care.

Eligible participants were provided with the participant's information sheet that details the purpose and nature of the study. All participants provided a written informed consent. Participants' ears were assessed by a qualified audiologist in one of the allocated rooms at the HIV clinic. Participants were only assessed if they were wearing face masks covering both the mouth and the nose and did not have COVID-19 related symptoms at the triage station. Each participant was sanitized before and after testing. The audiologist was also wearing personal protective equipment (PPE) in accordance with the national department of Health [31], which include N95 face mask, face shield, plastic apron and gloves during testing. The role of the audiologist was to capture the otoscopic images, save them in a folder for later analysis by the two otorhinolaryngologists.

Video otoscopy using Firefly Wireless DE550 was used to capture all images. Captured images were saved in one of the folders open specifically for this process. Upon 
completion of the assessment, all images were sent to the two otorhinolaryngologists via asynchronous tele-practice for analysis and diagnosis of middle ear integrity for possible pathologies [32]. The two otorhinolaryngologists were blinded on the demographic and medical characteristics of the participants. This method was used because it has been shown to have higher sensitivity and specificity in identifying middle ear pathologies [7, 33], it can be used where access to otorhinolaryngology services is limited or absent [34], and otorhinolaryngological analysis and diagnosis of the middle ear function and pathologies is considered a 'gold standard' in LMICs [35].

\section{Statistical Analysis}

Initially, all data were captured on an excel spreadsheet (office 365) before converting it into a software for analysis. The statistical software used was Stata version 15.2. Both descriptive and inferential statistics were used to analyze data. Descriptive statistics was used to describe and summarize the characteristics and some features of the data, including demography and middle ear functions of adults living with HIV. Frequencies and percentages for all categorical variables were used. Continuous variables were summarized using the median and interquartile range. In order to determine the reliability of the measure used, an inter-rater reliability of the two otorhinolaryngologists was assessed using the Cohen's Kappa test (k-value). Bivariate analysis to compare proportions was done using the Chisquare test.

\section{Results}

A total of 134 adults (268 ears) diagnosed with HIV were assessed. The diagnosis outcome from the two otorhinolaryngologists was classified into three categories: normal, abnormal, and could not evaluate (CNE). Where possible, otorhinolaryngologists provided the type of middle ear pathology observed on video otoscopy. There were more females $(64.18 \%, \mathrm{n}=86)$ than males $(35.82 \%, \mathrm{n}=48)$ in this study. The median age of the participants was 49 years with an interquartile range of 41-57 years. The median age differences were not significantly different between the two gender groups $(p$-value $=0.4367)$. Table 1 below summarizes the diagnoses made by each otorhinolaryngologist.

Regarding the left ear, majority of the participants had normal ears as determined by the two otorhinolaryngologists. There were $7.46 \%(n=10)$ participants with abnormalities from otorhinolaryngologist 1 and $4.48 \%(n=6)$ participants with abnormalities from the otorhinolaryngoloist2; however, this difference was not statistically significant. Regarding the right ear, majority of the participants also had normal ears. There were $5.57 \%$ $(\mathrm{n}=8)$ participants with abnormalities from the otorhinolaryngologist 1 and $4.48 \%(n=6)$ participants with abnormalities from otorhinolaryngologist2. There was no statistically significant difference between the proportions of these abnormalities in the right ear from the two otorhinolaryngologists. The significant association observed was mainly due to the absence of the wax outcome in the males which gave a $p$-value $=0.005$ of the otorhinolaryngologist 1 and $p$-value $=0.012$ for the otorhinolaryngologist2. Regarding both ears, majority of the participants had normal ears from video otoscopic findings. There were $6.72 \%(\mathrm{n}=18)$ participants with abnormalities from the otorhinolaryngologist 1 and $4.48 \%$ $(n=12)$ participants with abnormalities from the otorhinolaryngologist2. There was no significant difference between the proportions of these abnormalities from the two otorhinolaryngologists. The association of these abnormal outcomes and gender was marginal in the otorhinolaryngologist1 $(p$-value $=0.065)$ and statistically significant in the otorhinolaryngologist $2(p$-value $=0.017)$, with females presenting with more middle ear pathologies than males.

\section{Inter-rater Reliability of the Measures}

The two otorhinolaryngologists agreed on $78.49 \%$ $(n=205)$ of the total ears of patients reviewed (Table 2). The random agreement and perfect agreement of the two otorhinolaryngologists was estimated to have a Kapa value of $0.5801(58.01 \%)$. The amount of agreement indicates that the hypothesis that otorhinolaryngologists are making their determinations randomly can be rejected, $p$-value $<$ 0.001. Regarding the left ear only, the two otorhinolaryngologists agreed on $74.63 \%(n=100)$ of the left ears with the random agreement and perfect agreement estimated to have a Kapa value of 0.5569 (55.69\%). The amount of agreement was statistically significant, $p$ value $<0.001$. Regarding the right ear only, the two otorhinolaryngologists agreed on $78.36 \%(n=105)$ of the right ears with the random agreement and perfect agreement estimated to have a Kapa value of $0.6047(55.69 \%)$. The amount of agreement was statistically significant, $p$ value $<0.001$.

The most common abnormality detected by otorhinolaryngologist 1 was otitis media with effusion (OME) which accounted for $66.67 \%$ of the cases, while otorhinolaryngologist2 detected chronic suppurative otitis media (CSOM) which accounted for $58.33 \%$ of the total abnormalities detected. However, there was a poor diagnostic agreement $(\mathrm{k}=0.15)$ between the two otorhinolaryngologists based on the nature of the diagnosis. Figure 1 below provides a summary of the nature of abnormal middle ear 
Table 1 Diagnosis made otorhinolaryngologist

\begin{tabular}{|c|c|c|c|c|c|c|}
\hline & \multicolumn{3}{|c|}{ Otorhinolaryngologist1 } & \multicolumn{3}{|c|}{ Otorhinolaryngologist2 } \\
\hline & Female n (\%) & Male n (\%) & Total n (\%) & Female n $(\%)$ & Male n (\%) & Total n $(\%)$ \\
\hline \multicolumn{7}{|l|}{ Left ear diagnosis } \\
\hline Could not evaluate & $23(27.06)$ & $14(28.57)$ & $37(27.61)$ & $21(24.71)$ & $13(26.53)$ & $34(25.37)$ \\
\hline Abnormal & $7(8.24)$ & $3(6.12)$ & $10(7.46)$ & $4(4.71)$ & $2(4.08)$ & $6(4.48)$ \\
\hline Normal & $47(55.29)$ & $28(57.14)$ & $75(55.97)$ & $51(60.0)$ & $32(65.31)$ & $83(61.94)$ \\
\hline Wax & $8(9.41)$ & $4(8.16)$ & 12(8.96) & $9(10.59)$ & $2(4.08)$ & $11(8.21)$ \\
\hline Total & $85(100)$ & 49(100) & $134(100)$ & $85(100)$ & $49(100)$ & 134(100) \\
\hline Chi2 P-value & 0.963 & & & 0.612 & & \\
\hline \multicolumn{7}{|l|}{ Right ear diagnosis } \\
\hline Could not evaluate & $12(14.12)$ & $16(32.65)$ & $28(20.9)$ & $16(18.82)$ & $18(36.73)$ & $34(25.37)$ \\
\hline Abnormal & $5(5.88)$ & $3(6.12)$ & $8(5.97)$ & $4(4.71)$ & $2(4.08)$ & $6(4.48)$ \\
\hline Normal & $55(64.71)$ & $30(61.22)$ & $85(63.43)$ & $53(62.35)$ & $29(59.18)$ & $82(61.19)$ \\
\hline Wax & 13(15.29) & 0 & $13(9.7)$ & $12(14.12)$ & 0 & 12(8.96) \\
\hline Total & $85(100.0)$ & 49(100) & 134(100.0) & $85(100)$ & $49(100)$ & 134(100.0) \\
\hline Chi2 P-value & 0.005 & & & 0.012 & & \\
\hline \multicolumn{7}{|c|}{ Overall ears diagnosis } \\
\hline Could not evaluate & $35(20.59)$ & $30(30.61)$ & $65(24.25)$ & $37(21.76)$ & $31(31.63)$ & $68(25.37)$ \\
\hline Abnormal & $12(7.06)$ & $6(6.12)$ & $18(6.72)$ & $8(4.71)$ & $4(4.08)$ & $12(4.48)$ \\
\hline Normal & $102(60.0)$ & $58(59.18)$ & $160(59.7)$ & 104(61.18) & $61(62.24)$ & $165(61.57)$ \\
\hline Wax & $21(12.35)$ & $4(4.08)$ & $25(9.33)$ & $21(12.35)$ & $2(2.04)$ & $23(8.58)$ \\
\hline Total & $170(100)$ & $98(100)$ & $268(100)$ & $170(100)$ & $98(100)$ & $268(100)$ \\
\hline Chi2 P-value & 0.065 & & & 0.017 & & \\
\hline
\end{tabular}

The bold indicates significant of the number

Table 2 Agreement table of the two diagnostic tests

\begin{tabular}{|c|c|c|c|c|c|c|c|c|}
\hline $\begin{array}{l}\text { ENT2 } \\
\text { ENT1 }\end{array}$ & Could not evaluate & Abnormal & Normal & Wax & Total & Percentage agreement & Kappa value & P-value \\
\hline \multicolumn{9}{|c|}{ Overall test agreement } \\
\hline Could not evaluate & 40 & 6 & 16 & 3 & 65 & $78.49 \%(\mathrm{n}=205)$ & 0.5801 & $<0.0001$ \\
\hline Abnormal & 4 & 5 & 9 & 0 & 18 & & & \\
\hline Normal & 19 & 1 & 140 & 0 & 160 & & & \\
\hline Wax & 5 & 0 & 0 & 20 & 25 & & & \\
\hline Total & 68 & 12 & 165 & 23 & 268 & & & \\
\hline \multicolumn{9}{|l|}{ Left ear agreement } \\
\hline Could not evaluate & 21 & 3 & 11 & 2 & 37 & $74.63 \%(\mathrm{n}=100)$ & 0.5569 & $<0.001$ \\
\hline Abnormal & 2 & 3 & 5 & 0 & 10 & & & \\
\hline Normal & 8 & 0 & 67 & 0 & 75 & & & \\
\hline Wax & 3 & 0 & 0 & 9 & 12 & & & \\
\hline Total & 34 & 6 & 83 & 11 & 134 & & & \\
\hline \multicolumn{9}{|l|}{ Right ear agreement } \\
\hline Could not evaluate & 19 & 3 & 5 & 1 & 28 & $78.36 \%(\mathrm{n}=105)$ & 0.6047 & $<0.001$ \\
\hline Abnormal & 2 & 2 & 4 & 0 & 8 & & & \\
\hline Normal & 11 & 1 & 73 & 0 & 85 & & & \\
\hline Wax & 2 & 0 & 0 & 11 & 13 & & & \\
\hline Total & 34 & 6 & 82 & 12 & 134 & & & \\
\hline
\end{tabular}

The bold indicates significant of the number 
Fig. 1 Distribution of abnormalities as detected by each otorhinolaryngologist

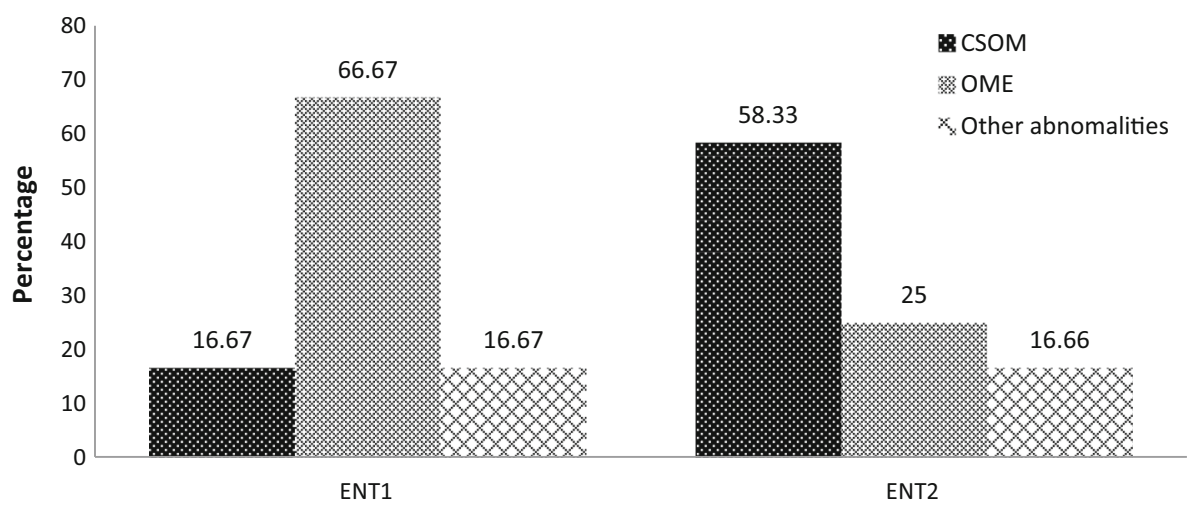

pathologies diagnosed by each of the participating otorhinolaryngologists.

\section{Discussion}

Despite the challenges that health care providers faced during initial implementation phases of tele-practice, the use of tele-practice has increased rapidly since COVID-19 outbreak [27]. One of the primary reasons for this exponential use of tele-practice was the need to reduce the risk of contracting and spreading COVID-19, particularly in patients who are at an increased risk for developing severe illnesses. Ohannesian [36] reported that tele-practice can be used in situations where healthcare facilities are under partial or complete quarantine, and therefore patients cannot access healthcare services.

This study investigated the use of tele-practice in assessing middle ear pathologies in adults living with HIV during COVID-19. Findings of this study demonstrate that tele-practice; in the form of asynchronous tele-audiology via video otoscopy can be used to assess middle ear function and pathologies in this population. Otorhinolaryngologists in this study were able to diagnose various middle ear pathologies. The most diagnosed middle ear pathologies in adults living with HIV in this study were OME and CSOM. These findings are consistent with previous research [7, 8, 37-39], indicating that OME and CSOM are common in adults living with HIV. Therefore, these findings support and call for widespread use of telepractice for assessment of middle ear pathologies in this population.

In this study, there were more females than male participants, with females presenting with significantly more middle ear pathologies than males. Tshifularo and colleagues [8] suggested that female predominance in research may be due to the fact that females tend to seek medical help earlier than men. While other studies on middle ear and HIV also reported that it is common for female participants to be significant more than males [3, 8, 38], these studies did not determine the effects of gender on middle ear function in adults living with HIV. In studies on HIV negative control group, results on the effects of gender on middle ear remains conflicting. Therefore, there is a need for large scale longitudinal studies to determine the effects of gender on middle ear pathologies in adults living with HIV.

Although the two otorhinolaryngologists were able to diagnose various middle ear pathologies through asynchronous tele-practice, the inter-rater reliability of the two otorhinolaryngologists, using Kappa statistics was low. For example, the overall agreement diagnoses for the abnormal results was considered moderate $(\mathrm{k}=0.58)$; while the agreement diagnoses for the nature of the abnormality (e.g. OME versus CSOM) was poor $(\mathrm{k}=0.15)$ [40]. These findings differ from previous research $[7,16]$, which found the agreement diagnoses between two independent otorhinolaryngologists were substantial $(\mathrm{k}=0.74$ and $\mathrm{k}=0.7$ respectively). The low inter-rater reliability between the two otorhinolaryngologists obtained in this current study may have been influenced by various factors.

There were a number of poor-quality images in the sample. Otorhinolarynogologist 1 could not evaluate $25.25 \%(n=65)$ of the ears, while otorhinolaryngologist 2 could not evaluate $25.3 \%(n=68)$ of the ears. In some cases, these number of poor-quality images were exacerbated by the presence of cerumen in the external ear canal (EAM). Cerumen impeded visualization of the tympanic membrane in $9.3 \%(n=25)$ and $8.6 \%(n=23)$ for the two otorhinolaryngologists respectively. The poor quality of images produced in this study may be due to the type of video otoscopy used. Research has already indicated that different video otoscopic instruments produce different quality images [41, 42]. In a study conducted by Sebothoma and Khoza-Shangase [7], an Otometrics Aurica Otocam 300 was used and it produced lower percentage (13\%) of poor-quality images. However, the current study used 
Firefly Wireless DE550, while a study by Biagio et al. [33] used a Welch Allyn digital MacroView Video otoscope.

These findings raise implications for the type and quality of tools and instruments used during asynchronous telepractice. The use of instruments that do not produce quality images prohibits clinicians who are not physically present with the patient to make appropriate diagnoses, especially early signs of middle ear pathologies. Current authors suspect that part of the reason less severe form of middle ear pathologies such as acute otitis media (AOM) were not identified in this population may be due to poor-quality images driven by the quality of the instrument used. In a study by Sebothoma and Khoza-Shangase [7] where a number of poor-quality images were lower (13\%), less severe middle ear pathologies were also commonly identified. It is therefore crucial that an instrument used during asynchronous tele-practice produces quality images in order to improve diagnoses and intervention, over and above training of site-facilitators on cerumen management to ensure that additional obstructions are removed before images are taken.

Ibekwe and Fasunla [43] suggested that given the shortages of otorhinolaryngologists in LMICs, there is a need to develop self-assessment applications that can be used for tele- otorhinolaryngology. These self-applications can be used by patients as part of the new technological development and task shifting paradigm to increase the use of tele-practice. English and colleagues [44] assert that COVID-19 challenges health systems to introduce new technology and change work patterns, including task shifting. Khoza-Shangase and Sebothoma [45] also argue for a task shifting paradigm in resource constrained environments as a method to reach those who do not have access to healthcare.

Instruments that produce quality images may not only be useful for tele-practice, but also for the new and emerging technology under the auspices of fourth industrial revolution (4IR). For example, emerging research has already explored the feasibility and applicability of using artificial intelligence (AI) based systems that can be used to identify middle ear pathologies [46, 47]. These AI based systems use image analysis classification of the tympanic membrane (TM) to identify middle ear pathologies. The accuracy of this system was found to have a higher sensitivity $(86.84 \%)$ in identifying patterns of middle ear pathologies. However, the successful use of these AI based systems will also require quality images in order to make appropriate diagnoses. Therefore, it is crucial to use instruments that produce quality images for early identification and intervention to be implemented.

\section{Limitation of the Study}

Although the current study provided some insights into the use of telepractice in the assessment of middle ear function in adults living with HIV during the COVID-19 pandemic, there are notable limitations. Firstly, the poor diagnostic agreement $(\mathrm{k}=0.15)$ between the two independent otorhinolaryngologists may have been due to poor quality of video otoscopic images captured during the assessment. These poor qualities of video otoscopic images raise important clinical implications. It is crucial that facilitators use equipment that captures quality video otoscopic images to enhance the diagnosis of middle ear pathologies. Although OME and CSOM are both considered to be more advanced forms of middle ear pathologies [11], these types of pathologies are different and require different interventions [49]. Therefore, misdiagnosing any of these pathologies may have dire consequences for the patient, hence the importance of good quality video capturing equipment, as well as comprehensive training of the site facilitators on how to properly capture clear video otoscopic images that can be used asynchronously for the diagnosis and subsequent intervention of middle ear pathologies. Lastly, this study utilized exclusively asynchronous telepractice, where otorhinolaryngologists independently assessed video otoscopic images and made the diagnoses of middle ear pathologies based solely on that, without conducting in-person assessments on the same sample. This is an acknowledged limitation for the study's ability to objectively compare and confirm the telepractice findings; however, due to COVID-19 this was not done. Although asynchronous telepractice has been found to be comparable with an in-person or onsite otomicroscopic diagnoses [16, 33], in person assessment by otorhinolaryngologists may still be required as gold standard assessment to mitigate the limitations created by asynchronous methods. Future studies, in the absence of the COVID-19 pandemic limitations, should utilize in-person assessment as well to compare the findings.

\section{Conclusion}

The findings of this study indicated that tele practice can be used to assess middle ear pathologies in adults living with HIV during COVID-19 pandemic. Asynchronous telepractice is particularly useful in countries where resources are limited and can assist patients to receive early identification and intervention. Given that the most diagnosed middle ear pathologies in this study were advanced or severe forms of middle ear pathologies such as CSOM, and the fact that the prevalence of HIV is high, current findings call for widespread use of tele-practice to identify early 
signs of middle ear pathologies. However, the success for asynchronous tele-practice in assessing middle ear pathologies in adults living with HIV during COVID-19 pandemic, is determined by the type of instrument used to capture quality images of the tympanic membrane, and professional training of otorhinolaryngologists on the use of this model of service delivery. In LMICs, where telepractice may continue beyond COVID-19 pandemic due to shortages of otorhinolaryngologists and access problems, the implications raised in this study are crucial and need to be addressed urgently.

Acknowledgements The researchers would like to acknowledge participants who volunteered to take part in the study

\section{Declarations}

Conflicts of interest The authors declare no conflicts of interest.

\section{References}

1. Joint United Nations program on AIDS/HIV. UNAIDS Data report 2018. 2018; available from: https://www.unaids.org/sit es/default/files/media_asset/unaids-data-2018_en.pdf: Accessed February 15, 2021

2. Joint United Nations program on AIDS/HIV. HIV and AIDS Estimates. 2020; Available from: unaids.org/en/regionscountries/countries/southafrica. Accessed February 15, 2021

3. Khoza K, Ross E (2002) Auditory function in a group of adults infected with HIV/AIDS in Gauteng. South Africa S Afr Commun Disord 49:17-27

4. Khoza-Shangase K (2011) An analysis of auditory manifestations in a group of adults with prior to antiretroviral therapy. Afr $\mathbf{J}$ infect Dis 51:11-22

5. Lin C, Lin SW, Weng SF, Lin YS (2013) Increased risk of sudden sensorineural hearing loss in patients with human immunodeficiency virus aged 18 to 35 years. JAMA Otolaryngol Head Neck Surg 139:251-255

6. Lugue AE, Orlando MS, Leong UC, Allen PD, Guido JJ, Yang H et al (2014) Hearing function in patients living with HIV/AIDS. Ear Hear 35:e282-e290

7. Sebothoma B, Khoza-Shangase K (2018) A comparison between video-otoscopy and standard tympanometry findings in Adults living with HIV in South Africa. S Afr Commun disord 65:1-7

8. Tshifularo M, Govender L, Monama G (2013) Otolaryngological and head and neck manifestations in HIV-infected patients seen at Steve Biko Academic Hospital in Pretoria. South Africa S Afr Med J 10:464-466

9. Sebothoma B, Khoza-Shangase K (2020) Middle ear pathologies in adults living with human immunodeficiency virus: a systematic review. Ann Otol Rhinol Laryngol 128:821-826

10. Vajpayee M, Negi N, Kurapati S (2013) The enduring tale of T cells in HIV immunopathogenesis. Indian $\mathrm{J}$ Med Res 138:682-699

11. Møller AR (2006) Hearing: anatomy, physiology, and disorders of the auditory system. Academic Press, San Diego

12. Kasemodel ALP, Costa LEM, Monsanto RC, Tomaz A, Penido NO (2020) Sensorineural hearing loss in the acute phase of single episode of acute otitis media. Braz J Otorhinolaryngol $86: 767-773$
13. Sharma N, Jaiswal AA, Banerjee PK, Garg AK (2015) Complications of chronic suppurative otitis media and their management: a single institution 12 Years' experience. Indian J Otolaryngol Head Neck Surg 67:353-360

14. World Health Organization. Addressing the rising prevalence of hearing loss. Available form: http://apps.who.int/iris/bitstre am/handle/10665/260336/9789241550260-eng.pdf;j sessionid=1 3FFE7CCEC3FE3335FD451FA5132CBD4? sequence $=1$. Accessed February 4, 2020

15. Sebothoma B, Khoza-Shangase K. Programmatic approach to prevent middle ear pathologies in South Africa: A proposed clinical framework. In Khoza-Shangase K, editor. Preventive Audiology: An African Perspective. Submitted

16. Biagio L, Swanepoel DW, Laurent C, Lundberg T (2014) Videootoscopy recordings for diagnosis of childhood ear disease using telehealth at primary health care level. J Telemed Telecare 20:300-306

17. Martin FN, Clark JG. Introduction to audiology. Edinburg: Pearson Education Limited. 2015.

18. Lu D, Wang H, Yu R, Yang H, Zhao Y (2020) Integrated infection control strategy to minimize nosocomial infection of coronavirus disease 2019 among ENT healthcare workers. J Hosp Infect 104:454-455

19. Canters for Disease Control and Prevention. COVID-19. Available from: https://www.cdc.gov/coronavirus/2019-ncov/ne ed-extra-precautions/people-with-medical-conditions.html?CDC_ AA_refVal=https $\% 3 \mathrm{~A} \% 2 \mathrm{~F} \% \quad 2 \mathrm{Fwww} . c d c . g o v \% 2 \mathrm{Fcoronavirus} \%$ 2F2019-ncov\%2Fneed-extra-precautions $\% 2$ Fgroups-at-higher-risk. html. Accessed January 30, 2021

20. Monaghesh E, Hajizedeh A (2020) The role of telehealth during COVID-19 outbreak: a systematic review based on current evidence. BMC Pub Health 20:1193. https://doi.org/10.1186/s12 889-020-09301-4

21. Reeves JJ, Ayers JW, Longhurst CA (2021) Telehealth in the COVID-Era: a balancing act to avoid harm. J Med Internet Res 23:e24785. https://doi.org/10.2196/24785

22. Swanepoel DW (2015) Tele-audiology. In: Katz J, English K, Tillery KL (eds) Handbook of clinical audiology. Wolters Kluver, Philadelphia, pp 659-672

23. Maurrasse SE, Rastatter JC, Hoff RS, Billings KR, Valika TS (2020) Telemedicine during the COVID-19 pandemic: a pediatric otolaryngology perspective. Otolaryngol Head Neck Surg 163:480-481

24. Ohlstein JF, Garner J, Takashima M (2020) Telemedicine in Otolaryngology in the COVID-19 Era. Initial Lesson Learn Laryngoscope 130:2568-2573

25. Aazh H, Swanepoel DW, Moore BCJ (2020) Telehealth tinnitus therapy during the COVID-19 outbreak in the UK: uptake and related factors. Int J Audiol. https://doi.org/10.1080/14992 027.2020 .1822553

26. Saunders GH, Roughly A (2020) Audiology in the time of COVID-19: practices and opinions of audiologists in the UK. Int J Audiol. https://doi.org/10.1080/14992027.2020.1814432

27. Doraiswamy S, Abraham A, Mamtani R, Cheema S (2020) Use of Telehealth during the COVID-19 pandemic: scoping review. J Med Internet Res 22:e24087. https://doi.org/10.2196/24087

28. Mulwafu W, Ensink R, Kuper H, Fagan J (2017) Survey of ENT services in sub-Saharan Africa: little progress between 2009 and 2015. Glob Health Action 10:1289736. https://doi.org/10.1 080/16549716.2017.1289736

29. Leedy PD, Ormrod JE (2013) Practical research: planning and design. Pearson Education, Boston

30. Hunter LL, Sanford CA (2015) Tympanometry and Wideband Acoustic Immittance. In: Katz J, English K, Tillery KL (eds) Handbook of Clinical Audiology. Wolters Kluver, Philadelphia, PA, pp 137-163 
31. Department of Health. COVID-19 Disease: infection Prevention and Control Guidelines version 2 ( $21^{\text {st }}$ May 2020). 2020. Available from: nicd.ac.za/wp-content/uploads/2020/05/ipc-guidelines-covid-19-version-2-21-may-2020.pdf. Accessed February 2, 2021

32. Hunter LL, Tubaugh L, Jackson A, Propos S (2008) Wideband middle ear power measurement in infants and children. J Am Acad Audiol 19:309-324

33. Biagio L, Swanepoel DW, Adeyemo A, Hall JW, Vinck B (2013) Asynchronous video-otoscopy with telehealth facilitator. Telemed J E Health 19:252-258

34. Metcalfe C, Muzaffar J, Orr L, Coulson C (2021) A systematic review of remote otological assessment using video-otoscopy over the past 10 years: reliability and application. Eur Arch Otorhinolaryngol. https://doi.org/10.1007/s00405-020-06596-2

35. Bright T, Mulwafu W, Phiri M, Ensink RJH, Smith A, Yip J et al (2019) Diagnostic accuracy of non-specialist versus specialist health workers in diagnosing hearing loss and ear disease in Malawi. Trop Med Int Health 24:817-828

36. Ohannessian R (2015) Telemedicine: potential applications in epidemic situations. Eur Res Telemed 4:65-98

37. Chandrasekar SS, Connelley PE, Brahmbhatt SS, Shah CS, Kloser PC, Beredes S (2000) Otologic and audiologic evaluation of human immunodeficiency virus-infected patients. AM J Otolaryngol 21:1-9

38. Obasineke G, Amdi FI, Ibekwe TS, Ezeanolue BC, Ogisi FO (2014) The effect of CD4 count level on the middle ear dynamics of HIV infected patients. East Afr Med J 91:29-32

39. Van der Westhuizen Y, Swanepoel DW, Heinze B, Hofmeyr LM (2012) Auditory and otological manifestation in adults with HIV/ AIDS. Int J Audiol 52:37-43

40. Viera AJ, Garrett JM (2005) Understanding interobserver agreement: the kappa statistic. Fam Med 37:360-363

41. Lundberg T, Westman G, Hellstrom S, Sandstrom H (2008) Digital imaging and telemedicine as a tool for studying inflammatory conditions in the middle ear evaluation of image quality and agreement between examiners. Int Pedatr Otorhinolaryngol 72:73-79. https://doi.org/10.1016/j.ijporl.2007.09.015

42. Mbao MN, Eikelboom RH, Atlas MD, Gallop MA (2003) Evaluation of video otoscopes suitable for tele-otology. Telemed J E Health 9:325-330

43. Ibekwe TS, Fasunla AJ (2020) Telemedicine in Otorhinolaryngological practice during COVID-19 pandemic. Niger Med J 61:111-113

44. English M, Moshabela M, Nzinga J, Barasa E, Tsofa B, Marchal $B$ et al (2020) Systems and implementation science should be part of the COVID-19 response in low resource settings. BMC Med 18:219. https://doi.org/10.1186/s12916-020-01696-6

45. Khoza-Shangase K, Sebothoma B. Tele-audiology and preventive audiology: A demand versus capacity challenge imperative in South Africa. In: Khoza-Shangase K, editor. Preventive Audiology: An African Perspective. Submitted

46. Myburgh HC, Van Zijl WH, Swanepoel DW, Hellström S, Laurent C (2016) Otitis media diagnosis for developing countries using tympanic membrane image-analysis. EBioMedicine 5:156-160

47. Myburgh HC, Jose S, Swanepoel DW, Laurent C (2018) Towards low-cost automated smartphone-and cloud-based otitis media diagnosis. Biomed Signal Process Control 39:34-52

48. World Health Organization. Diagnostic Errors. 2016; Available from: Apps.who.int/iris/bitstream/handle/10665/252410/978924 1511636-eng.pdf. Accessed February 20, 2021

49. Qureishi A, Lee Y, Belfield K, Birchall J, Daniel M (2014) Update on otitis media - prevention and treatment. Infect Drug Resist 7:15-24. https://doi.org/10.2147/IDR.S39637

Publisher's Note Springer Nature remains neutral with regard to jurisdictional claims in published maps and institutional affiliations. 